1997-01-01

\title{
Damped diocotron quasi-modes of non-neutral plasmas and inviscid fluids
}

\author{
S. Neil Rasband \\ judith@conselle.com \\ Ross L. Spencer \\ ross_spencer@byu.edu
}

Follow this and additional works at: https://scholarsarchive.byu.edu/facpub

Part of the Astrophysics and Astronomy Commons, and the Physics Commons

\section{Original Publication Citation}

Spencer, Ross L. and Neil S. Rasband."Damped diocotron quasi-modes of non-neutral plasmas and inviscid fluids." Physics of Plasmas 4 (1997): 53-6.

\section{BYU ScholarsArchive Citation}

Rasband, S. Neil and Spencer, Ross L., "Damped diocotron quasi-modes of non-neutral plasmas and inviscid fluids" (1997). Faculty Publications. 672.

https://scholarsarchive.byu.edu/facpub/672

This Peer-Reviewed Article is brought to you for free and open access by BYU ScholarsArchive. It has been accepted for inclusion in Faculty Publications by an authorized administrator of BYU ScholarsArchive. For more information, please contact ellen_amatangelo@byu.edu. 


\title{
Damped diocotron quasi-modes of non-neutral plasmas and inviscid fluids
}

\author{
Ross L. Spencer ${ }^{a)}$ and S. Neil Rasband \\ Department of Physics and Astronomy, Brigham Young University, Provo, Utah 84602
}

(Received 18 June 1996; accepted 30 September 1996)

\begin{abstract}
Computations of damped diocotron oscillations (quasi-modes) are described for non-neutral plasmas and inviscid fluids. The numerical method implements a suggestion made by Briggs, Daugherty, and Levy some 25 years ago [Phys. Fluids 13, 421 (1970)] to push the branch line that forms the continuum into the complex $\omega$-plane by solving the mode equation in the complex $r$-plane. For the special case of power-law density profiles the calculation finds the same quasi-mode frequencies found recently by Corngold [Phys. Plasmas 2, 620 (1995)]. It is found that the feature of the continuum eigenfunctions which indicates the presence of a nearby quasi-mode is continuity of the derivative of the regular part of the eigenfunctions near the singularity. The evolution of Rayleigh modes, found in density profiles with steps, is also studied as the density steps are smoothed. (C) 1997 American Institute of Physics. [S1070-664X(97)00801-X]
\end{abstract}

\section{INTRODUCTION}

The existence of a continuous spectrum and the possibility of damped oscillations for magnetized electron columns was discussed in an early paper by Briggs, Daugherty, and Levy, ${ }^{1}$ which was based on even earlier work by Case. ${ }^{2}$ These oscillations are connected to the continuous spectrum and their damping is called "spatial Landau damping" in Briggs et al. More recently these oscillations have been studied experimentally by Pillai and Gould ${ }^{3}$ and analytically (for the case of power-law equilibrium density profiles) by Corngold. ${ }^{4}$ (Corngold refers to these damped oscillations as "quasi-modes," a terminology that will be followed in this paper.) The continuation problem in the complex plane solved analytically by Corngold for power-law profiles can be solved numerically by implementing the suggestion of Briggs et al. to solve the radial mode equation along a contour in the complex $r$-plane ( $r$ is radius in cylindrical coordinates). Solving the mode equation along this complex $r$-contour moves the branch line (which corresponds to the continuum when the contour is real) into the complex $\omega$-plane, making it possible to "uncover" the damped quasimodes discussed by Briggs et al.

The diocotron mode equation and its general properties are discussed in Sec. II, the numerical method is described in Sec. III, and comparisons with Corngold's analytic results are described in Sec. IV. In Sec. V the behavior of quasimode frequencies as density profiles are changed from steplike to very broad is described. The behavior of the quasimode is tracked from its weakly damped beginnings where the analysis of Briggs et al. applies all the way to the parabolic quasi-mode of Corngold. In Sec. VI we explore the connection between quasi-mode frequencies and continuum eigenfunctions. It is found that for weak damping the real part of the quasi-mode frequency is close to the frequency of the continuum eigenfunction whose regular part near the singularity has a continuous first derivative. In Sec. VII the behavior of quasi-modes in density profiles with multiple steps is studied. We find that as such profiles are smoothed

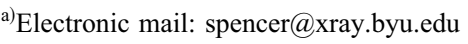

enough that there is no apparent remnant of the density steps, there are three different fates for the quasi-modes. They can either become highly damped and join the deformed branch line; they can become very weakly damped with resonant radii in a neighborhood of the center of the plasma if the density there is nearly constant; or two of them can both approach a common quasi-mode. In Sec. VIII we conclude the paper.

\section{MODE EQUATION}

Consider an infinitely long column of non-neutral plasma. The plasma is assumed to be so highly magnetized by a strong magnetic field in the $z$-direction that the Larmor radius is effectively zero, making it possible to model it as a charged fluid moving at the $\mathbf{E} \times \mathbf{B}$ drift velocity. Under these conditions the diocotron mode equation in cylindrical coordinates $(r, \theta)$ for perturbations proportional to $e^{i(m \theta-\omega t)}$ is

$$
\frac{1}{r} \frac{d}{d r} r \frac{d \phi}{d r}-\frac{m^{2} \phi}{r^{2}}-\frac{q n_{0}^{\prime}}{\epsilon_{0} B} \frac{m \phi}{r\left(\omega-m \omega_{0}(r)\right)}=0,
$$

where $\phi$ is the perturbed electrostatic potential; $q$ is the charge of the particles that make up the plasma; $\epsilon_{0}$ is the permittivity of free space; $B$ is the uniform axial magnetic field strength; $\omega_{0}$ is the $\mathbf{E} \times \mathbf{B}$ drift rotation frequency in the equilibrium radial electric field $\omega_{0}(r)=-E_{0} / r B$; and $n_{0}^{\prime}(r)$ is the radial derivative of the equilibrium density. The perturbed potential must be regular at the origin and vanish at $r=r_{w}$, the location of the conducting cylinder which surrounds the plasma. Note that Eq. (1) is also the mode equation for inviscid fluid motion, as pointed out by Briggs et al. Hence, all of the results discussed here also apply to inviscid fluids.

If the equilibrium density drops to zero rapidly in radius, leaving a sufficiently large vacuum region outside of the plasma, or if the density has discontinuous steps, then Eq. (1) will have solutions corresponding to undamped modes. The rest of the fluid dynamics described by this equation, however, is associated with the continuum, singular modes with frequencies that resonate with the equilibrium rotation: $\omega=m \omega_{0}(r)$ for values of $r$ where $n_{0}^{\prime}(r) \neq 0$. 
As pointed out by Briggs et al., if a step-like density profile having an undamped normal mode of frequency $\omega$ is smoothed so that there is a small amount of $n_{0}^{\prime}$ at the radius where $\omega=m \omega_{0}(r)$, then there are two ways to describe the new dynamics. Either we can say that the mode has merged with the continuum and has lost its identity, or we can say that it has been "pushed through" the continuum into the lower half of the complex $\omega$-plane, becoming a damped quasi-mode. [Note that having $\omega$ move down into the lower half-plane is opposite to the figures in Briggs et al. The reason is that their perturbations are proportional to $\exp (i \omega t)$ instead of to $\exp (-i \omega t)$, the convention in this paper.] This second description is similar to what happens in the case of Landau damping, and indeed this effect for diocotron modes is often referred to as "spatial Landau damping." As in Landau's calculation, special care must be taken to analytically continue the solutions into the lower half- $\omega$-plane.

Briggs et al. show how this continuation may be effected in their Figs. 6 and 9, where the interval in $r$ along which the mode equation is to be solved is deformed into the complex plane. The remainder of this paper is a description of what happens when this picture is taken seriously.

\section{NUMERICAL METHOD}

The quasi-mode may be "uncovered," as described by Briggs et al., by deforming into the complex plane the interval along which Eq. (1) is solved. The idea is to make the analytically-continued function $m \omega_{0}(r)$ describe a curve in the complex $\omega$-plane that dips sufficiently far into the lower half- $\omega$-plane. Since $m \omega_{0}(r)$ gives all the values of $\omega$ at which the mode equation is singular, this deformation pushes the branch line down into the lower half- $\omega$ plane, leaving the quasi-mode exposed above it. With the branch line out of the way it is then possible to calculate the quasi-mode frequency.

As an example, consider the family of power-law profiles discussed by Corngold: ${ }^{4}$

$$
\begin{aligned}
& n_{0}(r)=n_{00}\left[1-\left(\frac{r}{r_{w}}\right)^{2 p}\right] ; \\
& \omega_{0}(r)=\omega_{00}\left[1-\frac{1}{1+p}\left(\frac{r}{r_{w}}\right)^{2 p}\right] ; \quad \omega_{00}=-\frac{q n_{00}}{2 \epsilon_{0} B},
\end{aligned}
$$

where $p$ takes on integer values $1,2,3, \ldots, r_{w}$ is the radius of the conducting cylinder that surrounds the plasma, and $n_{00}$ is the central density. Substitution of complex values of $r / r_{w}$ into the expression for $\omega_{0}(r)$ shows that to make $\omega_{0}(r)$ dip into the lower half of the $\omega$-plane the $r$-contour must be pushed up into the upper half of the complex $r$-plane.

This deformation may be made numerically by changing the independent variable from $r$ to $s$ according to

$$
\begin{aligned}
& r=r_{w}[s+i h(s)], \\
& h(0)=0 ; \quad h(1)=0 ; \quad h(s) \geqslant 0 ; \quad 0 \leqslant s \leqslant 1,
\end{aligned}
$$

where $r_{w}$ is the radius of the conducting cylinder that surrounds the plasma.

The numerical procedure consists of rewriting Eq. (1) in terms of $s$ instead of $r$, subdividing the $s$-interval $[0,1]$ into small subintervals. The resulting differential equation is solved in two different ways. The first is to make finitedifference approximations to the derivatives that appear in the transformed mode equation. The second is to expand $\phi$ in terms of cubic splines and to use a Galerkin approximation to the differential equation. These two different ways of calculating have provided a check on the numerical results presented here. In either case a homogeneous system of equations is obtained which is solved by matrix shooting. ${ }^{5}$ This simply means that the numerical approximation to the mode equation at one of the grid points in $s$ is removed and replaced by the equation $\phi=1$; then the resulting inhomogeneous system is solved. The mode frequency $\omega$ is then varied until the equation that was removed from the system is satisfied. If $h(s)$, the imaginary part of $r(s)$, is large enough and properly shaped, this algorithm finds quasi-modes.

The analytic continuations of some density profiles, especially those with sharp gradients, often have places in the complex $r$-plane where they become large or change rapidly, making it difficult to compute efficiently. In such cases it is sometimes helpful to shape the $r$-contour so that it only has a large imaginary part near the radius where the quasi-mode is located. Practical experience has shown that the following form for $h(s)$ is usually sufficiently flexible to find quasimodes:

$$
h(s)=4 h_{0} s(1-s) e^{-\left(s-s_{0}\right)^{2} / w^{2}},
$$

where $h_{0}$ is a parameter that determines the height of the $r$-contour. The parameters $s_{0}$ and $w$ make a Gaussian multiplier that can be used to make $r(s)$ nearly real except near $r\left(s_{0}\right)$, making it possible to avoid troublesome places in the complex $r$-plane.

\section{COMPARISON WITH ANALYTIC CALCULATIONS}

Corngold ${ }^{4}$ studied the power-law profiles given in Eq. (2), showing that the mode equation could be put in the form of the hypergeometric differential equation. This makes it relatively easy to effect the required analytic continuation of $\phi(r)$ into the complex $r$-plane. An exact calculation like this is precisely what is needed to verify that the numerical method described above is working, but Corngold does not give exact results; he emphasizes the qualitative behavior of the quasi-mode frequencies as functions of mode number $m$ and density profile parameter $p$. Moreover, there are two misprints in his paper that must be corrected in order to see that the numerical method described here agrees with his analytic treatment. Hence, it is necessary to briefly review his calculation.

The exact dispersion relation for the quasi-mode frequencies in Corngold's calculation is

$$
\begin{aligned}
& F(\overline{a, b ;} \overline{a+} \overline{b+1} ; z)-2 \pi i \frac{\Gamma(\overline{a+} \overline{b+1})}{\Gamma(\bar{a}) \Gamma(\bar{b})}(1-z) F(\overline{a+1} 1, \bar{b} \\
& \quad+1 ; 2 ; 1-z)=0,
\end{aligned}
$$

where $F$ is the hypergeometric function. The quasi-mode frequency $\omega$ is related to the solution $z$ of this equation by 


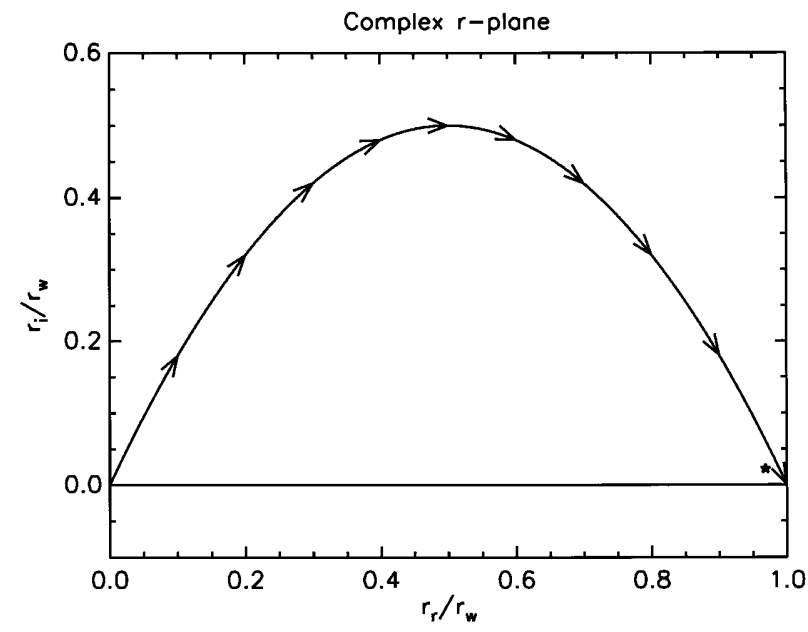

FIG. 1. A contour in the complex $r$-plane which uncovers the damped quasi-mode for a parabolic density profile is shown. The arrowheads indicate the direction of increasing contour parameter $s$, with the arrowheads equally spaced in $s$. This contour has $h_{0}=0.5, s_{0}=0.5$, and $w=10^{6}$ [meaning that the Gaussian in $h(t)$ is essentially unity]. The star shows the place in the complex $r$-plane where the quasi-mode lies, i.e., the complex value of $r$ where $m \omega_{0}(r)$ is equal to the complex frequency of the quasi-mode.

$$
\omega=m \omega_{00} \frac{(1+p) z-1}{(1+p) z},
$$

and the parameters $\bar{a}$ and $\bar{b}$ are given by

$$
\begin{aligned}
& \bar{a}=\frac{m}{2 p}-\sqrt{\left(\frac{m}{2 p}\right)^{2}+\frac{p+1}{p}} ; \\
& \bar{b}=\frac{m}{2 p}+\sqrt{\left(\frac{m}{2 p}\right)^{2}+\frac{p+1}{p}} .
\end{aligned}
$$

In Corngold's paper the factor $2 \pi i$ in Eq. (5) is given with a positive sign, but $-2 \pi i$ as given above is correct. ${ }^{6}$

As a first check the numerical procedure was applied to the $m=2$ mode of the parabolic density profile used by Corngold $(p=1)$ with contour parameters $h_{0}=0.5$, $s_{0}=0.5, w=10^{6}$ (effectively setting the Gaussian to 1 ), and with 5000 subintervals. This contour is shown in Fig. 1, while Fig. 2 shows the corresponding contour in the complex $\omega$-plane of the function $m \omega_{0}(r)$, the deformed branch-line contour. Using the numerical procedure of Sec. III, a quasimode was found at $\omega / \omega_{00}=1.06249648-i 0.04260298$ while the corresponding solution of Eq. (5) is $\omega / \omega_{00}$ $=1.06249647-i 0.04260304$. This quasi-mode is also indicated by the stars in Figs. 1 and 2. (In Sec. III of Corngold's paper the frequency of this mode is given as $\omega=0.94+i 0.046$, but this is also a misprint. The approximate procedure outlined in Sec. II of Corngold's paper actually gives $\omega=1.06-i 0.04{ }^{6}$ ) Other comparisons of Eq. (5) with matrix shooting along a complex $r$-contour for other choices of $m$ and $p$ give similar accuracy, indicating that the numerical method works quite well.

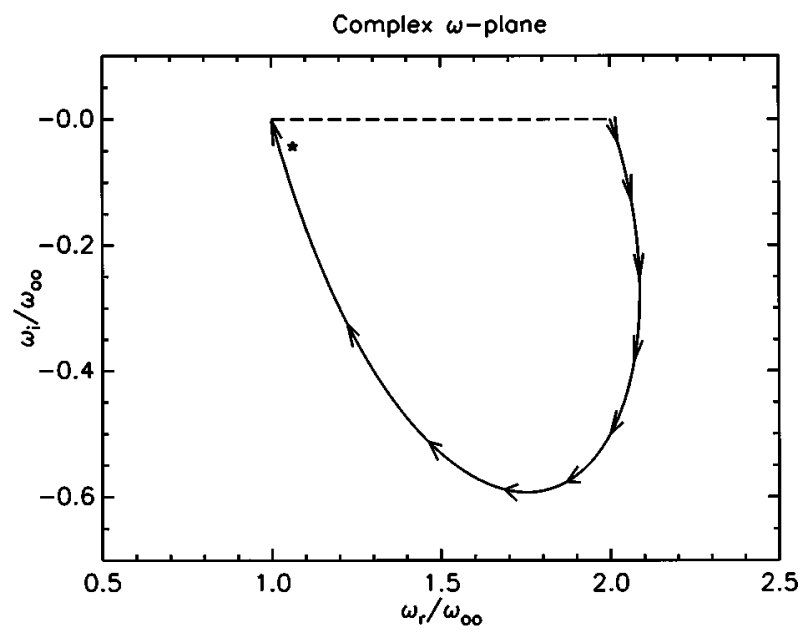

FIG. 2. A contour in the complex $\omega$-plane which uncovers the damped quasi-mode for a parabolic density profile is shown. This contour is the deformed position of the branch line $m \omega_{0}(r)$ corresponding to the $r$-contour shown in Fig. 1. The arrowheads indicate the direction of increasing contour parameter $s$, with the arrowheads equally spaced in $s$. The star shows the place in the complex $\omega$-plane where the quasi-mode lies. The dashed line shows the position of the branch line (continuum) before the $r$-contour was deformed.

\section{TRANSITION FROM STEP TO PARABOLIC}

Briggs et al. discuss the case of a density profile which is a sharp step with constant density $n_{00}$ for $r \leqslant r_{p}$ and zero density for $r_{p}<r \leqslant r_{w}$. In this case there is no continuum and a single undamped mode with frequency,

$$
\omega=\omega_{00}\left[m-1+\left(r_{p} / r_{w}\right)^{2 m}\right] .
$$

They then show that as the sharp edge of this density profile is smoothed, putting a small amount of density at the radius where the frequency of Eq. (8) is the same as $m \omega_{0}(r)$, the undamped mode becomes a damped quasi-mode. Their approximate procedure is not powerful enough, however, to investigate what happens to the quasi-mode when the density profile is smoothed enough that the density at the resonant radius is no longer small.

To study this problem a form for the density profile must be chosen. A simple choice would be the power-law profile studied by Corngold [Eq. (2)], for as $p$ approaches infinity these profiles approach a sharp step. However, if the density goes to zero at the wall, as it does in the profile given in Eq. (2), then we are restricted to cases where there is no vacuum region outside the plasma. And if we modify these profiles so that there is a vacuum region (Corngold's "gap" profiles ${ }^{4}$ ) then the density profile is not analytic across the entire radius and we would have to modify our numerical method. It could be done, but it is awkward, so we consider instead the following analytic form for the equilibrium density $n_{0}(r)$ :

$$
n_{0}(r)=n_{00} \frac{\left[\tanh k\left(r_{w}^{2}-C\right)-\tanh k\left(r^{2}-C\right)\right]}{\left[\tanh k\left(r_{w}^{2}-C\right)+\tanh k C\right]},
$$

or (equivalently) 


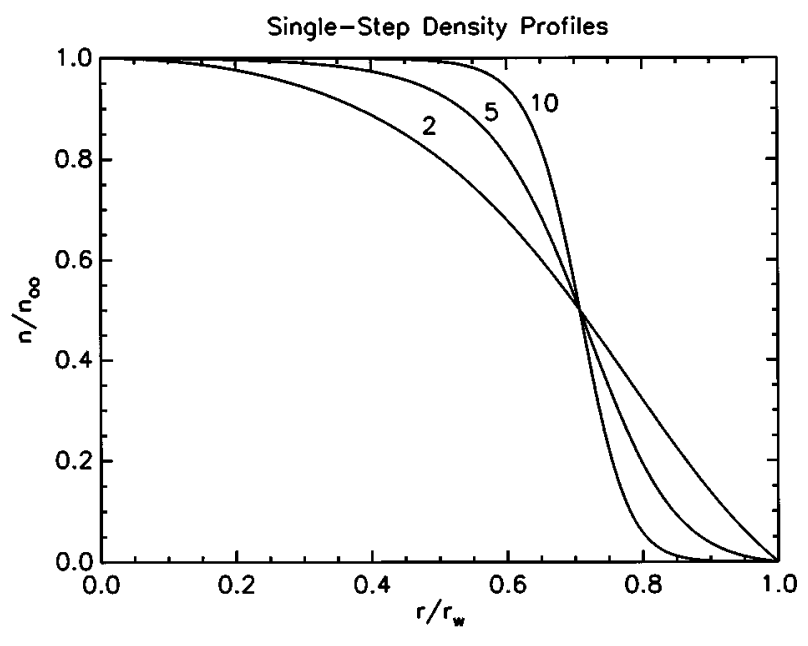

FIG. 3. Three density profiles that interpolate between a sharp step and a parabola are shown. The values of $k r_{w}^{2}$ used in Eq. (11) to give these profiles are indicated just above each profile.

$$
n_{0}(r)=n_{00} \frac{\left[1-\exp \left(-2 k\left(r_{w}^{2}-r^{2}\right)\right)\right][1+\exp (-2 k C)]}{\left[1+\exp \left(2 k\left(r^{2}-C\right)\right)\right]\left[1-\exp \left(-2 k r_{w}^{2}\right)\right]},
$$

where

$$
k C=\frac{1}{2} \ln \left(\frac{2}{\epsilon}-1\right)
$$

and

$$
\begin{aligned}
\epsilon= & 2 e^{-2 k r_{p}^{2}} \\
& \times \frac{1-2 \exp \left(-2 k\left(r_{w}^{2}-r_{p}^{2}\right)\right)+\exp \left(-2 k r_{w}^{2}\right)}{1-\exp \left(-2 k r_{p}^{2}\right)-\exp \left(-2 k r_{w}^{2}\right)+\exp \left(-2 k\left(r_{w}^{2}+r_{p}^{2}\right)\right)} .
\end{aligned}
$$

The form of the density function given in Eq. (9) is chosen to represent either sharp steps or broad transitions, depending on the value chosen for $k$. The equivalent form in Eq. (10) and the formula for $\epsilon$ in Eq. (11) are built to avoid the numerical overflow problems that arise when using the tanh-function directly. The density at the plasma radius $r_{p}$ is always $n_{00} / 2$ and the parameter $k$ governs the sharpness of the density step. The density is constrained to be zero at $r=r_{w}$. When $k$ is large the step is sharp and as $k$ is reduced the step becomes broadened. The only choice of $r_{p}$ that allows $k$ to become arbitrarily small is $r_{p} / r_{w}=1 / \sqrt{2}$; all other choices for $r_{p}$ have a minimum value of $k$ below which $k C$ becomes imaginary and the functional form no longer works.

Figure 3 shows what these density profiles look like for three different values of $k r_{w}^{2}$, all with $r_{p} / r_{w}=1 / \sqrt{2}$. For this special choice of $r_{p}$, as $k \rightarrow 0$ the density profile becomes parabolic. Because $\tanh z$ is analytic, except at isolated points along the imaginary axis, the density function of Eq. (9) can be continued into the complex plane to search for the quasi-mode associated with the undamped mode that is found when the step is infinitely sharp.

Figure 4 shows the result of matrix shooting to deter-

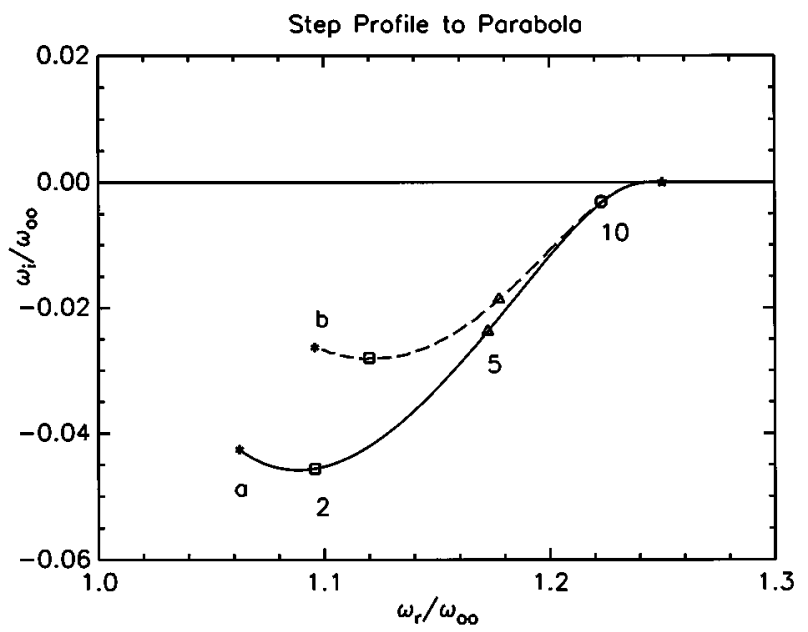

FIG. 4. (a) The path of the $m=2$ quasi-mode frequency is shown as the density profile is changed from a sharp step (the star at $\omega / \omega_{00}=1.25$ ) to a parabola (the asterisk at $\omega=1.06-i 0.043$ ). The three symbols along the curve correspond to the three density profiles shown in Fig. 3. The numerical values $(2,5,10)$ associated with the symbols correspond to the $k r_{w}^{2}$-values indicated in Fig. 3. (b) The approximate path of the $m=2$ quasimode frequency is shown for the same profile variation as in curve (a). Along this curve $\omega_{r}$ was obtained from the $b_{+}=b_{-}$condition on the continuum eigenfunction and $\omega_{i}$ was obtained from the approximate Briggs formula [their Eq. (50)].

mine $\omega$ with $r_{p}$ fixed at $r_{p} / r_{w}=1 / \sqrt{2}$ and for values of $k r_{w}^{2}$ ranging from 20 (sharp step) down to 0.03 (nearly a parabolic density profile). The solid curve gives the variation of the quasi-mode frequency as it changes from the sharpedge value (the star on the right end of the curve) to the parabolic value (the ${ }^{*}$ on the left end). Note that many different density profiles were used to make this curve in Fig. 4, not just the three profiles shown in Fig. 3.

\section{ESTIMATING THE QUASI-MODE FREQUENCY FROM THE CONTINUUM}

One of the mysteries of the damped quasi-mode is what determines its frequency, $\omega_{r}$. In an earlier study of these quasi-modes deGrassie and Malmberg used a numerical technique to ignore the resonance in Eq. (1) ${ }^{7}$ and got good quantitative agreement with experimentally observed quasimode frequencies, but it seems qualitatively that this frequency should somehow be connected to the continuum modes. But of all the frequencies in the continuum, what is special about the frequency of the quasi-mode? To see the connection of the quasi-mode frequency with the continuum we must first recall what the continuum eigenmodes look like. They are singular solutions of Eq. (1) with $\omega$ belonging to the range of real frequencies that make the resonant denominator in the equation vanish. At the singular radius $r_{s}$ the perturbed potential has a finite value, but its derivatives are singular. Near the singular point the leading terms in the expansion of $\phi$ obtained from the usual Frobenius analysis are

$$
\phi \approx \phi\left(r_{s}\right)\left(1-\frac{q n_{0}^{\prime}\left(r_{s}\right)}{\epsilon_{0} B r_{s} \omega_{0}^{\prime}\left(r_{s}\right)} x \ln |x|\right)+b_{ \pm} x,
$$


where $x=r-r_{s}$. The coefficient $b_{ \pm}$of the regular part of the solution actually represents two coefficients, $b_{+}$for the solution when $x$ is positive and $b_{-}$when $x$ is negative. In general these two coefficients are not equal, but they might be. And, in fact, the continuum frequency at which they are equal gives a good estimate of the quasi-mode frequency.

To see why, consider the limit in which $n_{0}^{\prime}\left(r_{s}\right)$ becomes vanishingly small. In this limit the logarithmic singularity in Eq. (12) becomes quite insignificant, so the continuum eigenfunctions are mostly characterized by the mismatch between $b_{+}$and $b_{-}$. Since the damped quasi-mode is very close to a real undamped mode in this limit, and since the real mode has continuous derivatives, the only continuum eigenmode which can become the real eigenmode of the point spectrum is the special one with $b_{+}=b_{-}$.

These coefficients are found by numerically computing the continuum eigenfunctions and comparing the solutions near the singular point with the approximation in Eq. (12) to determine $b_{ \pm}$. This approximation technique has been compared with exact results from Corngold's calculation ${ }^{4}$ for many combinations of $m$ and $p$, and it is found that the relative error in the determination of $\omega_{r}$ is of order $\omega_{i} / \omega_{r}$. When $\omega_{i}$ is large, however, this continuum approximation becomes increasingly poor, then fails altogether. The reason is that modes with large imaginary part may not be resonant in the plasma at all, as pointed out by Corngold. ${ }^{4}$

It is also possible to estimate the damping rate by combining the estimate of $\omega_{r}$ given above with the approximate formula for $\omega_{i}$ given by Briggs et al. [their Eq. (50)]:

$$
\frac{\omega_{i}}{\omega_{00}}=\frac{\pi r_{p} n_{0}^{\prime}\left(r_{s}\right)}{2 m n_{00}}\left(\frac{r_{p}}{r_{s}}\right)^{2 m-3}\left[1-\left(\frac{r_{s}}{r_{w}}\right)^{2 m}\right]^{2}
$$

where $r_{s}$ is the resonant radius, i.e., $m \omega_{0}\left(r_{s}\right)=\omega_{r}$. In doing this calculation the resonant radius was taken to be the resonant radius of the continuum eigenfunction approximation to the quasi-mode (the one for which $b_{+}=b_{-}$). Once $r_{s}$ was determined in this way the actual value of $n_{0}\left(r_{s}\right)$ for the diffuse density profile was used. The dashed curve in Fig. 4 shows the result of this approximate calculation on the same equilibrium sequence used for the solid curve. Note that for small $\omega_{i}$ the approximate calculation works very well. But for very broad profiles this is a rather crude estimate since Eq. (13) is based on a step-like density profile. Indeed, as can be seen from Fig. 4, it only predicts $\omega_{i}$ within about a factor of 2 for such profiles.

More elaborate approximation methods based on the continuum eigenfunctions have also been tried, but these have always turned out to be quite poor and even more difficult to carry out than the correct numerical calculation along the complex $r$-contour.

\section{DENSITY PROFILES WITH MULTIPLE STEPS}

Briggs, Daugherty, and Levy ${ }^{1}$ also discuss the problem of multiple steps in the density profile. In the case of several sharp steps, Rayleigh ${ }^{8}$ showed that there is a discrete mode associated with each step. Briggs et al. showed that as each step is smoothed slightly each of these modes becomes a damped quasi-mode. In this section the question of what

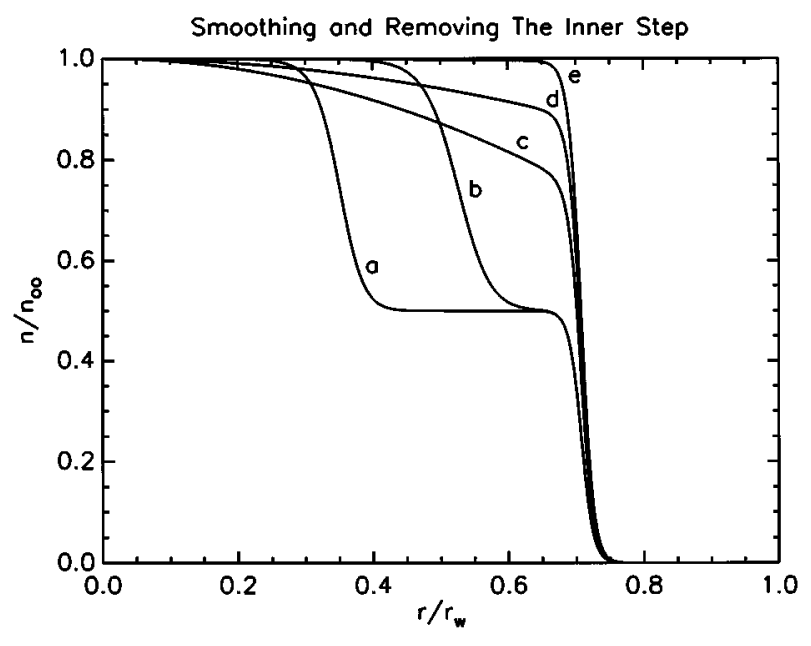

FIG. 5. A sequence of density profiles is shown which modifies a doublestep profile $(a)$ into a single-step profile $(e)$. Both steps of the profile are made by using the hyperbolic tangent form of a smooth step discussed in Sec. V. The transition from $(a)$ to $(c)$ is made by reducing $k$ for the inner step while keeping the size of the step constant. The final transition from $(c)$ to $(e)$ is made by keeping the inner $k$ constant while reducing the size of the step to zero.

happens to these quasi-modes as changes are made to the density profile will be addressed. First to be discussed is the case of a double-step profile in which the outer step is kept sharp while the inner one is first smoothed, then reduced in size to approach a single-step profile. Discussed in the second part of this section is the case in which a double-step profile is smoothed so that the end result is a parabolic profile. In both cases the double-step profile is made by combining two of the hyperbolic-tangent profiles discussed in Sec. V. Finally, the problem of power-law profiles with vacuum gaps discussed by Corngold ${ }^{4}$ will be discussed and it will be shown that for most gap widths there is an additional undamped mode present in addition to the damped quasimode discussed in Ref. 4.

\section{A. Double-step to single-step}

Consider the sequence of density profiles shown in Fig. 5. The sequence $a-c$ involves smoothing the inner step by reducing its $k$-value while increasing the radius at which the step occurs. Figure 6 shows what happens to the frequency of the quasi-mode associated with the inner step as this transformation is made. As expected from the analysis of Briggs et al., it acquires a negative imaginary part. (The quasi-mode associated with the outer step remains resonant and essentially undamped in the space between the plasma and the wall during all of these changes. This rather uninteresting behavior is not shown.)

The second sequence $c-e$ is made by keeping the $k$-value of the inner step constant while reducing its size. This sequence is interesting because the final profile (curve $e$ in Fig. 5) is a single step, which is expected to have only a single quasi-mode, resonant between the plasma and the wall; so what is to become of the inner quasi-mode?

The answer for this case is shown by the dashed path in Fig. 6. Its damping rate goes to zero and its real frequency approaches $m \omega_{00}$, indicating at first glance that this mode 


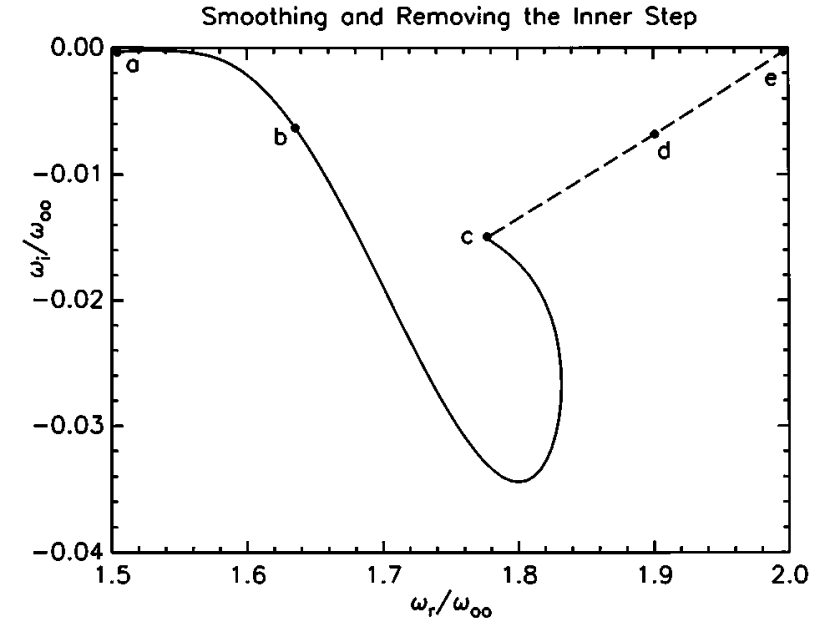

FIG. 6. The behavior of the $m=2$ quasi-mode frequency associated with the inner step of the double-step profile $(a)$ in Fig. 5 is shown. At $e$ the damping rate is nearly 0 while $\omega_{r} \approx m \omega_{00}$. Even though $\omega_{i}$ is small (about $3 \times 10^{-4}$ ) and $\omega_{r} \approx m \omega_{00}$, however, the resonant radius in the complex $r$-plane is $r / r_{w}=0.62+i 0.023$.

should be resonant at $r=0$. This is not the case, however. The numerical calculation gives a resonant radius for the entire sequence from $c$ to $e$ that varies between $0.66 r_{w}$ and $0.62 r_{w}$, while the imaginary part of the complex resonant $r$ is nearly constant at $r_{i}=0.023 r_{w}$. This apparent discrepancy between the sizes of $\omega_{i}$ and $r_{i}$ is resolved by noticing that for small $r_{i}$ they are related by

$$
\omega_{i} \approx m r_{i} \frac{d}{d r} \omega_{0}(r) .
$$

Hence, if the $\omega_{0}$-profile is nearly constant, as it is inside the plasma for profile $e$ in Fig. 5, the damping rate can be very small even though $r_{i}$ is finite. (The eigenfunction along the real- $r$ axis for this quasi-mode would have a rather large discontinuity because of the logarithmic branch cut extending down to the real axis from $r / r_{w}=0.62+i 0.023$, as illustrated in Fig. 8 of Briggs et al. $)^{1}$

So density profiles that look very much like a single-step might actually have several quasi-modes: the usual one resonant outside the the plasma, as discussed by Briggs et al., and one or more quasi-modes resonant inside the plasma corresponding to small wrinkles in the density profile. Calculations of this kind have also been done for triple-step profiles where it is found that there can be two interior quasimodes as a single-step profile is approached, provided that the triple-step profile structure is still present, albeit with vanishingly small size. These quasi-modes are the remnants of Rayleigh's discrete modes, but because they all have very small damping rates and frequencies very near $m \omega_{0}(0)$, it is not clear that they have any dynamical importance.

\section{B. Double-step to parabola}

Consider now the sequence of density profiles shown in Fig. 7. In this case the final parabolic profile is one for which we know there is only one quasi-mode, raising the question of how the transition is made from the two quasi-modes of

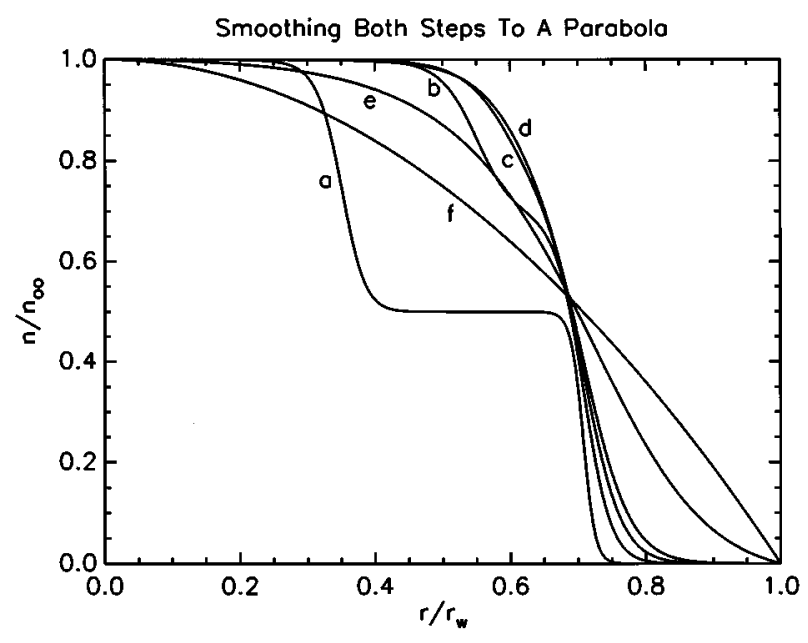

FIG. 7. A sequence of density profiles is shown which modifies a doublestep profile $(a)$ into a parabola $(f)$. Both steps of the profile are made by using the hyperbolic tangent form of a smooth step discussed in Sec. V. Profiles $c$ and $d$, though very nearly the same, have quite different quasimode frequencies for the quasi-mode associated with the inner step (see Fig. 8).

profile $a$ to the single one of profile $f$. The answer is shown in Fig. 8. The dashed curve shows what happens to the quasimode which is resonant in the space between the plasma and the conducting wall. As the profile changes from a doublestep to a parabola, it simply makes its way toward the quasi-mode frequency of a parabolic profile $\omega / \omega_{00}$ $=1.0625-i 0.0426$.

The solid curve shows what happens to the inner quasimode. As expected, it becomes more damped as the profile is smoothed, but in the neighborhood of profiles $c$ and $d$ in Fig.

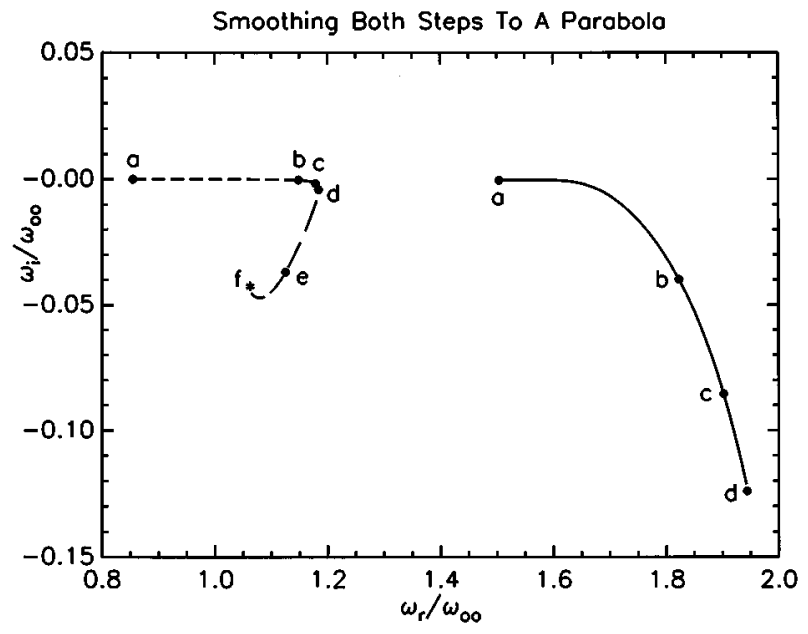

FIG. 8. The behavior of both of the $m=2$ quasi-mode frequencies associated with the two steps of the double-step profile $(a)$ in Fig. 7 is shown. The solid curve shows the behavior of the quasi-mode associated with the inner step. Notice that the slight profile change between $c$ and $d$ (see Fig. 7) corresponds to a large change in $\omega_{i}$ for this mode. Indeed, the quasi-mode cannot be tracked further because it has rushed downward to join the deformed branch line. The dashed curve shows the behavior of the quasi-mode associated with the outer step. Its frequency varies smoothly between the double-step value $(a)$ and the parabolic value $(f)$ where $\omega / \omega_{00}=1.0625-i 0.0426$. 
7 the imaginary part rapidly becomes larger in magnitude. It becomes so large, in fact, that it is not possible to move the deformed branch line contour ahead of it without encountering wild places in the complex $\omega$-plane, and it joins the branch line. This is the reason that no points beyond $d$ are indicated on its path. So in this case one of the quasi-modes is eliminated by having it join the (deformed) branch line.

\section{Parabola with a gap to a single-step}

The reviewer of this paper asked a question and made an interesting observation, both of which will be addressed here. The question does not involve density profiles with multiple steps, but it is related to the question of what happens to two quasi-modes when the profile is changed to one that has only one, so it seems best to discuss it here. The question is whether it is not also possible to approach a single-step by increasing $p$ in Corngold's profiles [see Eq. (2)]. It is, of course, possible to do it this way, but if the density vanishes at the conducting wall then the single-step just fills the entire region, which is not very interesting. And if the density goes to zero at a radius less than $r_{w}$, then there is a vacuum gap (as discussed by Corngold), but the density profile is non-analytic which is awkward to study using the numerical methods discussed here. But thinking about approaching a single-step in this way leads to the interesting observation made by the reviewer.

The observation is that there is a qualitative discrepancy between Corngold's discussion of the spectrum when a gap is present and the results presented here. In Corngold's gap calculation $^{4}$ there is a single quasimode whose frequency approaches the mode frequency of the single-step as $p$ is increased without bound. But in the case of profile $c$ in Fig. 5 there are two quasi-modes: the first is the one described by the curve in Fig. 6 and the second is one that is resonant out in the exponentially small density tail so that it is practically undamped. This second one is the one whose frequency approaches the mode frequency of the single-step as the density profile goes through the sequence $c$ to $d$ to $e$ in Fig. 5. But profile $c$ in Fig. 5 does not look much different from a power-law profile with a vacuum gap, so why do we find two quasi-modes while Corngold only finds one?

The answer to this question is that there is a subtle point in the power-law profile calculation that was missed in Ref. 4. This point is illustrated by Fig. 9. The solid circles in frame (a) of Fig. 9 show the variation of quasimode frequency with gap width using Corngold's dispersion function, but instead of setting it to zero it is required to satisfy the gap boundary condition, Corngold's Eq. (23):

$$
\psi+\frac{p}{m}\left[1-\left(\frac{r_{p}}{r_{w}}\right)^{2 m}\right] \frac{d \psi}{d z}=0,
$$

where $\psi(z)$ is the function given on the left-hand side of Eq. (5). The solid circles were obtained by using the secant method and a standard hypergeometric function subroutine to solve Eq. (15), and they agree very well with the data given in Fig. 7 of Ref. 4. Note, however, that our Fig. 9 covers a larger range of gaps than does the figure in Corngold's paper (his data correspond to $\omega_{r} / \omega_{00}>0.8$ in Fig. 9).
Complex Frequency vs Gap, Quodratic Profile, $m=2$
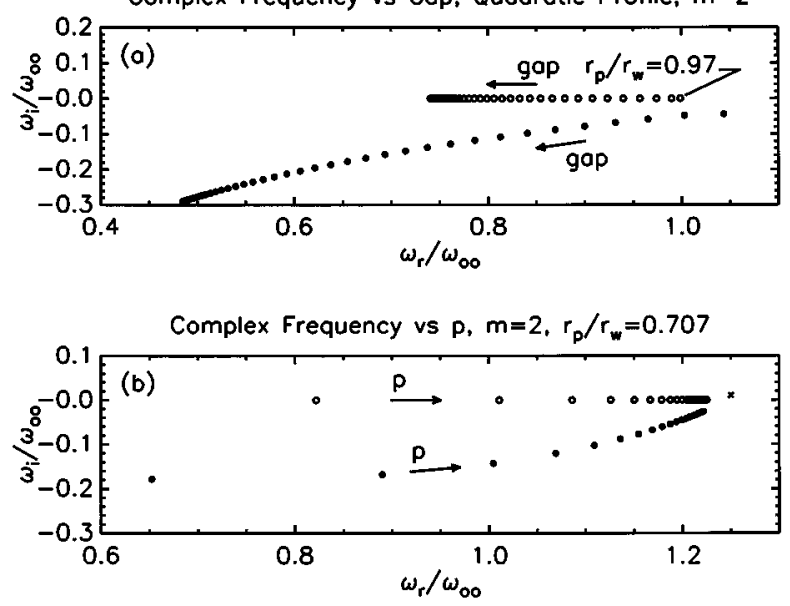

FIG. 9. (a) Shown here is the behavior of the quasi-mode and mode frequencies of a parabolic density profile as the vacuum gap is increased. Fifty different equally spaced gaps ranging from $r_{p} / r_{w}=0.99$ down to $r_{p} / r_{w}=0.01$ were used. The solid circles show the 50 damped quasi-mode frequencies corresponding to the analytically continued sheet of Corngold. The open circles show the undamped mode frequencies that correspond to the original sheet. There are no such modes until $r_{p} / r_{w}<0.97$. (b) The behavior of the quasi-mode and undamped mode for $r_{p} / r_{w}=0.707$ as $p$ is increased is shown. As the density profile approaches a single-step profile $(p \rightarrow \infty)$ both converge to the undamped mode frequency of a the single step at $\omega / \omega_{00}=1.25$ (indicated by the symbol $\times$ ).

But in addition to the quasi-mode solution of Eq. (15) discussed in Ref. 4, there is also an undamped mode for most gap widths. This mode may be obtained simply by not performing the analytic continuation that gives the extra term containing $2 \pi i$ in Eq. (5), working instead with the original function $\psi(z)=F(\overline{a, b} ; \bar{a}+\bar{b}+1 ; z)$. When there is no gap no modes are obtained from this sheet of $\psi(z)$, as noted by Corngold. But as the gap is widened, at about $r_{p} / r_{w}=0.97 \mathrm{a}$ mode appears. This undamped mode is resonant in the vacuum gap and its variation with gap width is shown by the open circles in frame (a) of Fig. 9. Similar calcultions with other values of mode number $m$ and profile parameter $p$ show that as $p$ increases the critical value of $r_{p} / r_{w}$ approaches 1 [for $m=2$ and $p=(1,2,3)$ the critical values are $\left.r_{p} / r_{w}=(0.97,0.98,0.99)\right]$ and as $m$ increases the critical value decreases, but not by much. For example, for $p=1$ and $m=8$ an undamped mode exists for $r_{p} / r_{w}<0.9$. Hence, for most power-law profiles with gaps (and particularly for profiles with gaps as big as the one in Fig. 5) there are both a damped quasi-mode and an undamped mode.

Now we may explore what happens to the quasi-mode and the undamped mode as $p$ is increased, approaching a single-step profile. The answer to this question is shown in frame (b) of Fig. 9 where, once again, the quasi-mode is indicated by solid circles and the undamped mode is indicated by open circles. Note that both frequencies converge to the single undamped mode of the single-step profile (indicated by the symbol $\times$ in the figure). This is yet another scenario for what happens when a profile that has two quasimodes is changed to one that only has one: both may converge to the same location.

So here are three scenarios for what happens when a 
profile that has two quasi-modes is changed into one that has only one: (1) one can disappear by joining the deformed branch line (Figs. 7 and 8), (2) one can disappear by approaching $m \omega_{0}$ while being resonant at a finite radius (Figs. 5 and 6), or (3) the two can both converge to the one quasimode of the final profile (Fig. 9b). The numerical experiments we have performed indicate that these three possibilities are common, but we are now sufficiently humbled by the complexity of these problems that we would not be surprised if something else were also possible.

\section{CONCLUSION}

Damped diocotron quasi-modes have been computed by using standard shooting techniques along contours in the complex $r$-plane, implementing a suggestion made by Briggs, Daugherty, and Levy. ${ }^{1}$ This numerical technique agrees with the analytic dispersion relation found by Corngold, ${ }^{4}$ but can handle a much wider variety of density profiles. We also find an answer to the question, "Of all the frequencies in the continuum, what is special about the frequency of the damped quasi-mode?' We find that the frequency of the quasi-mode matches the frequency of the con- tinuum eigenfunction whose regular part has a continuous first derivative across the singularity. Finally, these computations have been used to learn what happens to quasi-modes as density profiles are changed from one form into another. The ability to compute these quasi-modes and understand their behavior as density profiles are modified should be helpful in understanding their significance in the dynamics of non-neutral plasmas and inviscid fluids.

\section{ACKNOWLEDGMENTS}

The authors are grateful for useful discussions with Noel Corngold and with Ann Cass.

${ }^{1}$ R. J. Briggs, J. D. Daugherty, and R. H. Levy, Phys. Fluids 13, 421 (1970).

${ }^{2}$ K. M. Case, Phys. Fluids 3, 143 (1960).

${ }^{3}$ N. S. Pillai and R. W. Gould, Phys. Rev. Lett. 73, 2849 (1994).

${ }^{4}$ N. R. Corngold, Phys. Plasmas 2, 620 (1995).

${ }^{5}$ J. P. Freidberg and D. W. Hewett, J. Plasma Phys. 26, 177 (1981).

${ }^{6}$ N. R. Corngold (private communication, 1996).

${ }^{7}$ J. S. deGrassie and J. H. Malmberg, Phys. Rev. Lett. 39, 1077 (1977); Phys. Fluids 23, 63 (1980).

${ }^{8}$ J. W. S. Rayleigh, Proc. London Math. Soc. 11, 57 (1880). 